\title{
SIMULTANEOUS EFFECTS OF HEAT TRANSFER AND VARIABLE VISCOSITY ON PERISTALTIC TRANSPORT OF CASSON FLUID FLOW IN AN INCLINED POROUS TUBE
}

\author{
G. MANJUNATHA and C. RAJASHEKHAR \\ Department of Mathematics, Manipal Institute of Technology \\ Manipal Academy of Higher Education \\ Manipal, Karnataka, INDIA \\ H. VAIDYA* \\ Department of Mathematics, SSA Government First Grade College (Autonomous) \\ Ballari, Karnataka, INDIA \\ E-mail: hanumeshvaidya@gmail.com \\ K.V. PRASAD \\ Department of Mathematics, Vijayanagara Srikrishnadevaraya University \\ Ballari, Karnataka, INDIA
}

\begin{abstract}
The present study investigates the combined effects of varying viscosity and heat transfer on a Casson fluid through an inclined porous axisymmetric tube in the presence of slip effects. The modeled governing equations are solved analytically by considering the long wavelength and small Reynolds number approximations. The numerical integration is employed to obtain pressure rise and frictional force. A parametric analysis has been presented to study the effects of the Darcy number, angle of inclination, varying viscosity, velocity slip, thermal slip, yield stress, amplitude ratio, Prandtl number and Eckert number on the pressure rise, pressure gradient, streamlines, frictional force and temperature. The study reveals that an increase in the angle of inclination and viscosity parameter has a proportional increase in the pressure rise. Also, an increase in the porosity causes a significant reduction in the pressure rise.
\end{abstract}

Key words: Darcy number, thermal slip, velocity slip, variable viscosity, yield stress.

\section{Introduction}

The study of flow through a porous medium has acquired a considerable interest of researchers in recent time due to its applications in understanding various mechanisms in lungs, gallbladder, blood vessel movement, etc. In the human body, a large part of the muscle is a porous structure. These structures are essential to supply the nutrients to every cell, and their proper functioning mainly depends upon the blood flowing through them. In such situations, the presence of slip on the boundary due to the porosity of the wall plays an essential role in inspecting the flow of blood in arteries. Thus, slip effects are more articulated for fluids moving through geometries which have flexible property, like blood vessels. The experimental investigations on blood flow revealed the significance of slip at the porous walls. Specifically, peristaltic movement of blood through a tube can be modeled better by taking slip and porosity into account. The preliminary investigation on peristaltic transport was initially carried out by Latham [1]. Subsequently, many

\footnotetext{
* To whom correspondence should be addressed
} 
researchers have carried out theoretical and experimental studies on peristaltic transport by taking different geometries and assumptions (Burns and Parkes [2]; Shapiro et al. [3]; Rajashekhar et al. [4]). Early literature on the peristaltic mechanism suggests that the study on the porosity of the wall has not been given much importance it deserves. Taking this fact into consideration, the first attempt was made by Elshehawey et al. [5]. Later, Elshehawey and Husseny [6] studied peristaltic transport through a porous medium bounded by two porous plates. Vajravelu et al. [7] used the Phan-Thein-Tanner fluid to study the peristaltic movement. The study on MHD Couette flow of a Jeffery fluid in a porous medium was investigated by Sreenadh et al. [8]. Sankad and Nagathan [9] carried out the studies on peristaltic transport of a couple stress fluid under the influence of slip and heat transfer in a porous medium. Recently, Ellahi et al. [10], investigated peristaltic transport of a nanofluid by considering entropy generation and porous medium. Most of the studies on peristaltic transport are carried out by taking Newtonian or non-Newtonian approach. This approach may be sufficient to understand the urine flow through the ureter and flow of blood in large arteries where shear rate is high, but it fails to explain the complex rheological behavior of blood in narrow arteries where the shear rates are low. Studies on a non-Newtonian nature of blood flow have been of most importance to researchers in recent years due to their application in investigating the behavior of blood in narrow arteries. Casson fluid is one of such non-Newtonian fluids which exhibits yield stress and is adequate for the description of the flowing blood when the shear rates are low. Blair [11] observed that at low shear rates, the Casson model was more accurate in predicting the physiological behaviors of blood. Thus, numerous researchers studied of the Casson model under different physiological fluids (Nagarani [12]; Vajravelu et al. [13]; Prasad et al. [14]; Vajravelu et al. [15]). Apart from the Casson model, the Herschel-Bulkley model also includes yield stress and studies concerning the use of the model on peristaltic transport were reported in the studies of (Chaturani and Narasimhan [16]; Manjunatha et al. [17]; Manjunatha and Rajashekhar [18]).

The above mentioned studies do not explain the heat transfer effects on peristaltic transport. The study of heat transfer effects along with slip conditions on peristalsis has attracted attention of researchers due to the extensive application in the field of biofluid mechanics, chemical engineering, and medicine. Several researchers examined the interaction between peristalsis and heat transfer in different geometries with and without slip conditions. By considering the elastic nature of the tube Radhakrishnamacharya and Srinivasulu [19] explored peristaltic transport with the effects of heat transfer. Later, Srinivas and Kothandapani [20] investigated the impact of heat transfer for peristaltic transport in an asymmetric channel. Nadeem and Akbar [21] analyzed the effects of different rheological parameters on the pressure rise, frictional force, and temperature. Vajravelu et al. [22] examined the effects of heat transfer and permeability of the tube on peristaltic transport by using a Jeffery model. Subsequently, numerous researchers investigated the effect of heat transfer on peristaltic transport in different geometries (Vajravelu et al. [23]; Ramesh and Devakar [24]; Hayat et al. [25]; Ebaid et al. [26]; Vaidya et al. [27]; Devaki et al. [28]).

Most of the investigations on peristaltic transport have been done by taking the viscosity of the fluid to be. This supposition fails to give a better understanding of the peristaltic mechanism involved in lymphatic vessel, intestine, blood flow in small arteries, etc. In these organs viscosity of the fluid varies across the thickness of the fluid (Hayat and Ali [29]; Lachiheb [30]; Awais et al. [31]). Thus, considering variable viscosity helps in understanding peristaltic transport of non-Newtonian fluids in the above-mentioned organs.

To the best of authors' knowledge, no attempts have been made in the literature to investigate the combined effects of slip, heat transfer and inclination on peristaltic transport of a Casson fluid in an axisymmetric porous tube with variable viscosity. The present investigation is helpful in filling the gap in this area. The flow is assumed to be steady, fully developed and laminar. The closed-form solutions are obtained for velocity, flow rate, pressure, streamline, pressure rise and frictional force. Further, the impact of the amplitude ratio, yield stress, Darcy number, velocity slip parameter, angle of inclination, thermal slip parameter, viscosity parameter, Eckert number, Prandtl number on pressure, streamline, pressure rise, frictional force and temperature are presented graphically by using MATLAB.

\section{Mathematical formulation}

Consider peristaltic transport of an incompressible viscous fluid with effects of variable viscosity, 
slip and heat transfer in an axisymmetric inclined porous tube of radius $a$ (Fig.1) and inclined at an angle $\beta$ to the horizontal. The wall deformation due to the propagation of an infinite sinusoidal wave with constant speed $c$ along the walls of the tube is given by the following equation.

$$
h(z, t)=1+\varepsilon \sin \left[\frac{2 \pi}{\lambda}(z-c t)\right]
$$

where $\varepsilon$ is the amplitude ratio, $\lambda$ is the wavelength, $c$ is the wave speed, $t$ is the time and $z$ is the axial direction.

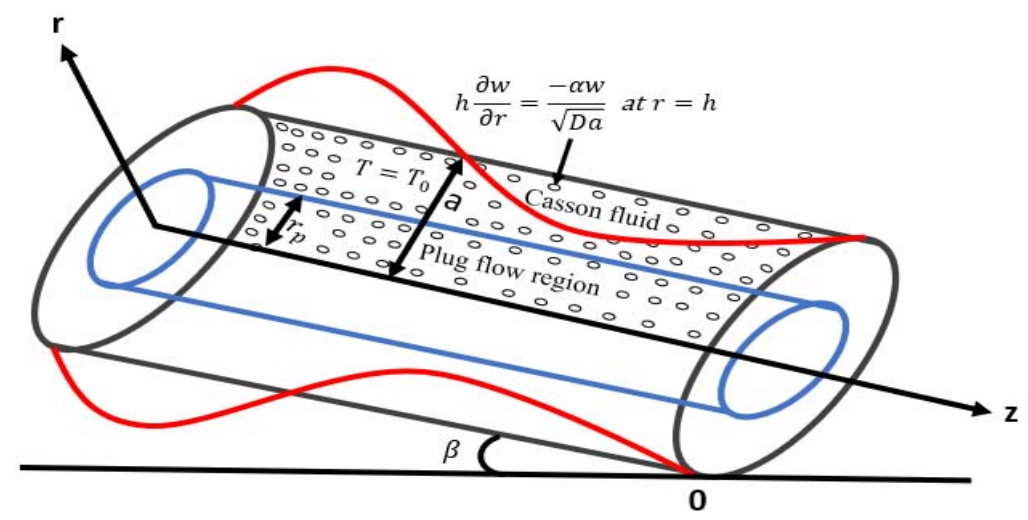

Fig.1. Geometrical representation of peristaltic waves.

\section{Mathematical modelling and solutions to the problem}

The equations of motion and energy in the wave frame of reference, moving with speed $c$, under the lubrication approach (Nadeem and Akbar [21]) are as follows

$$
\begin{aligned}
& \operatorname{Re} \delta\left(u \frac{\partial}{\partial r}+w \frac{\partial}{\partial z}\right) w=-\frac{\partial p}{\partial z}+\frac{1}{r} \frac{\partial}{\partial r}\left(r \tau_{r z}\right)+\delta \frac{\partial}{\partial r}\left(\tau_{z z}\right), \\
& \operatorname{Re} \delta^{3}\left(u \frac{\partial}{\partial r}+w \frac{\partial}{\partial z}\right) u=-\frac{\partial p}{\partial r}+\frac{\delta}{r} \frac{\partial}{\partial r}\left(r \tau_{r r}\right)+\delta^{2} \frac{\partial}{\partial r}\left(\tau_{r z}\right), \\
& \operatorname{Re} \delta \operatorname{Pr}\left(u \frac{\partial}{\partial r}+w \frac{\partial}{\partial z}\right) \theta=\operatorname{Ec} \operatorname{Pr}\left(\delta \frac{\partial u}{\partial r} \tau_{r r}+\frac{\partial w}{\partial r} \tau_{r z}+\delta^{2} \frac{\partial u}{\partial r} \tau_{z r}+\tau_{z z} \frac{\partial w}{\partial r} \delta\right)+ \\
& +\frac{\partial^{2} \theta}{\partial r^{2}}+\frac{1}{r} \frac{\partial \theta}{\partial r}+\delta^{2} \frac{\partial^{2} \theta}{\partial z^{2}}
\end{aligned}
$$

where $u$ and $w$ are the radial and axial velocities, $\operatorname{Re}$ is the Reynolds number, $\theta$ is temperature, $\delta$ is the wave number, $\mathrm{Pr}$ is the Prandtl number, Ec is the Eckert number, $r$ is the radial coordinate, $\tau_{r r}$ is the shear stress in radial coordinates $\tau_{z r}$ is the shear stress in axial and radial coordinates, $\tau_{z z}$ is the shear stress in axial coordinate and $\tau_{r z}$ is the shear stress along radial and axial coordinates.

The following nondimensional variables are introduced: 


$$
\begin{aligned}
& \bar{r}=\frac{r}{a}, \quad \bar{z}=\frac{z}{\lambda}, \quad \bar{t}=\frac{c t}{\lambda}, \quad \bar{\tau}_{0}=\frac{\tau_{0}}{\mu_{0}\left(\frac{c}{a}\right)}, \quad \bar{\tau}_{r z}=\frac{\tau_{r z}}{\mu_{0}\left(\frac{c}{a}\right)}, \quad \bar{p}=\frac{p a^{2}}{\lambda c \mu_{0}}, \\
& \bar{r}_{p}=\frac{r_{p}}{c}, \quad \varepsilon=\frac{b}{a}, \quad \operatorname{Pr}=\frac{\mu_{0} c_{p}}{k}, \quad \bar{u}=\frac{u}{c}, \quad \bar{w}=\frac{w}{c}, \quad \theta=\frac{T-T_{0}}{T_{0}}, \\
& \mathrm{Ec}=\frac{c^{2}}{c_{p} T_{0}}, \quad \delta=\frac{a}{\lambda}, \quad \bar{\tau}_{a}=\frac{\tau_{a}}{\mu_{0}\left(\frac{c}{a}\right)}, \quad \operatorname{Re}=\frac{\rho c a \delta}{\mu_{0}}, \quad F_{l}=\frac{\mu_{0} c}{\rho g a^{2}}, \quad \bar{\mu}(r)=\frac{\mu(\mathrm{r})}{\mu_{0}} .
\end{aligned}
$$

Under the assumption of long wavelength $\delta<<1$ and small Reynolds number $(\operatorname{Re} \approx 0)$, Eqs (3.1)(3.3) takes the form

$$
\begin{aligned}
& \frac{1}{r} \frac{\partial}{\partial r}(r \tau)=-\frac{\partial p}{\partial z}+\frac{\sin \beta}{F_{1}}, \\
& 0=\frac{\partial p}{\partial r}, \\
& \frac{1}{r} \frac{\partial}{\partial r}\left(r \frac{\partial \theta}{\partial r}\right)=\operatorname{Ec} \operatorname{Pr}\left(-\frac{\partial w}{\partial r} \tau\right) .
\end{aligned}
$$

The constitutive equation for Casson's fluid in the non-dimensional form is given by Blair [11]

$$
\begin{aligned}
& \frac{\partial w}{\partial r}=-\left(\frac{1}{\mu(r)}\left[\sqrt{\tau_{r z}}-\sqrt{\tau_{0}}\right]\right)^{2}, \quad \tau_{r z} \geq \tau_{0}, \\
& \frac{\partial w}{\partial r}=0, \quad \tau_{r z} \leq \tau_{0} .
\end{aligned}
$$

The corresponding non-dimensional boundary conditions are

$$
\begin{aligned}
& h \frac{\partial w}{\partial r}=\frac{-\alpha w}{\sqrt{\mathrm{Da}}}, \quad \theta+\gamma \frac{\partial \theta}{\partial r}=0 \quad \text { at } \quad r=h, \\
& \frac{\partial \theta}{\partial r}=0, \tau_{r z} \quad \text { is finite at } \quad r=0 .
\end{aligned}
$$

Equation (3.10) corresponds to the velocity and thermal slip conditions, respectively [32]. Further, $\mathrm{Da}$ is the porous parameter (Darcy number), $\alpha$ is the velocity slip parameter, $\gamma$ is the thermal slip parameter and $\theta$ is the temperature.

The influence of variation in viscosity is chosen in the following form 


$$
\mu(r, z)=e^{-\alpha_{1} r}, \quad \text { for } \quad 0 \leq r \leq h
$$

or

$$
\mu(r, z)=1-\alpha_{1} r, \quad \text { for } \quad \alpha_{1}<<1
$$

where $\alpha_{1}$ is the viscosity parameter. The choice of $\alpha_{1}$ here is reasonable physiologically because a typical individual or creature of comparable size takes 1-2 L of fluid every day. Additionally, 6-7 L is produced by the digestive tract as discharges from salivary organs, stomach, pancreas, liver, and the small digestive system itself. This incorporates the reliance of fluid concentration upon the radial axis, which influences the viscosity to diminish nearer to the vessel membrane.

Solving Eqs (3.5) and (3.6) with the conditions (3.10), (3.11) and (3.13), we obtain

$$
\begin{aligned}
& w=\frac{P+f}{2}\left[\frac { 1 } { \alpha _ { 1 } ^ { 2 } } \left\{a_{1}(r-h)-4 \alpha_{1}\left(\sqrt{r r_{p}}-\sqrt{h r_{p}}\right)+4 \sqrt{a_{1} r_{p}}\left(\tanh ^{-1} \sqrt{a_{1} r}-\tanh ^{-1} \sqrt{a_{1} h}\right)+\right.\right. \\
& \left.\left.+\left(1+\alpha_{1} r_{p}\right) \log \left(\frac{1-a_{1} r}{1-a_{1} h}\right)\right\}+\frac{h \sqrt{\mathrm{Da}}}{\alpha\left(1-\alpha_{1} h\right)}\left(h+r_{p}-\sqrt{h r_{p}}\right)\right]
\end{aligned}
$$

where $\quad P=-\frac{\partial p}{\partial z}$ and $f=\frac{\sin \beta}{F_{1}}$.

Using the condition $\tau_{0}=\frac{\operatorname{Pr}_{p}}{2}$ at $r=r_{p}$, the upper limit of the plug flow region is obtained as $r_{p}=\frac{2 \tau_{0}}{P}$. Also, by using the condition $\tau_{r z}=\tau_{h}$ at $r=h$ (Bird et al. [33]), we obtain

$$
P=\frac{2 \tau_{h}}{h}
$$

Hence,

$$
\frac{r_{p}}{h}=\frac{\tau_{0}}{\tau_{h}}=\tau .
$$

Using relation (3.16) and taking $r=r_{p}$ in Eq.(3.14), we obtain the plug flow velocity as

$$
\begin{aligned}
& w_{p}=\frac{P+f}{2}\left[\frac { 1 } { \alpha _ { 1 } ^ { 2 } } \left\{a_{1}\left(r_{p}-h\right)-4 \alpha_{1}\left(r_{p}-\sqrt{h r_{p}}\right)+4 \sqrt{a_{1} r_{p}}\left(\tanh ^{-1} \sqrt{a_{1} r_{p}}-\tanh ^{-1} \sqrt{a_{1} h}\right)+\right.\right. \\
& \left.\left.+\left(1+\alpha_{1} r_{p}\right) \log \left(\frac{1-a_{1} r_{p}}{1-a_{1} h}\right)\right\}+\frac{h \sqrt{\mathrm{Da}}}{\alpha\left(1-\alpha_{1} h\right)}\left(h+r_{p}-\sqrt{h r_{p}}\right)\right] .
\end{aligned}
$$

Using Eq.(3.7) together with the boundary conditions (3.10) and (3.11), we obtain an expression for the temperature profile as 


$$
\begin{aligned}
& \theta=(P+f)^{2} \operatorname{Ec} \operatorname{Pr}\left[\frac{r^{4}}{64}+\frac{r_{p} r^{3}}{36}-\frac{2}{49} r_{p}^{1 / 2} r^{7 / 2}-\frac{h^{4}}{64}+\right. \\
& \left.-\frac{r_{p} h^{3}}{36}+\frac{2}{49} r_{p}^{1 / 2} h^{7 / 2}-\gamma\left(\frac{h^{4}}{16}+\frac{r_{p} h^{3}}{36}-\frac{1}{7} r_{p}^{1 / 2} h^{7 / 2}\right)\right] .
\end{aligned}
$$

Integrating Eqs (3.14) and (3.17) and using the conditions $\psi_{p}=0$ at $r=0$ and $\psi=\psi_{p}$ at $r=r_{p}$, the stream function for $r_{p} \leq r \leq h$ is given by

$$
\begin{aligned}
& \psi=\frac{P+f}{12 \alpha_{1}^{3}}\left[R_{1}+\frac{1}{\alpha\left(\alpha_{1} h-1\right)}\left\{R_{2}+\alpha\left(\alpha_{1} h-1\right)\left[R_{3}+\alpha_{1}\left(R_{4}+r_{p} R_{5}\right)\right]+\right.\right. \\
& \left.\left.+3 \alpha\left(\alpha_{1} h-1\right)\left[R_{6}+R_{7}+\left(1+\alpha_{1} r_{p}\right)\left(2 R_{8}+R_{9}\right)\right]\right\}\right]
\end{aligned}
$$

and the stream function for the plug flow in the region $0 \leq r \leq r_{p}$ can be written as

$$
\begin{aligned}
& \psi_{p}=\frac{(P+f) r_{p}^{2}}{4}\left[\frac { 1 } { \alpha _ { 1 } ^ { 2 } } \left\{a_{1}\left(r_{p}-h\right)-4 \alpha_{1}\left(r_{p}-\sqrt{h r_{p}}\right)+4 \sqrt{a_{1} r_{p}}\left(\tanh ^{-1} \sqrt{a_{1} r_{p}}+\right.\right.\right. \\
& \left.\left.\left.-\tanh ^{-1} \sqrt{a_{1} h}\right)+\left(1+\alpha_{1} r_{p}\right) \log \left(\frac{1-a_{1} r_{p}}{1-a_{1} h}\right)\right\}+\frac{h \sqrt{\mathrm{Da}}}{\alpha\left(1-\alpha_{1} h\right.}\left(h+r_{p}-\sqrt{h r_{p}}\right)\right] .
\end{aligned}
$$

The instantaneous volumetric flow rate in the wave frame is given by

$$
\begin{aligned}
& Q=2\left[\int_{0}^{r_{p}} w_{p} r d r+\int_{r_{p}}^{h} w r d r\right], \\
& Q=\frac{P+f}{60 \alpha}\left[R_{10}\left(1+\alpha_{1} h\right)+\frac{R_{11}}{\alpha_{1}^{4}}+\frac{R_{12}}{\alpha_{1}^{3}}+\frac{R_{13}}{\alpha_{1}^{\frac{7}{2}}}+\frac{R_{14}}{\alpha_{1}^{2}}+\frac{R_{15}}{\alpha_{1}}+R_{16}\right]+O\left(\alpha_{1}^{2}\right) .
\end{aligned}
$$

Thus, we have

$$
\frac{\partial p}{\partial z}=f-60 \alpha Q\left[R_{10}\left(1+\alpha_{1} h\right)+\frac{R_{11}}{\alpha_{1}^{4}}+\frac{R_{12}}{\alpha_{1}^{3}}+\frac{R_{13}}{\alpha_{1}^{\frac{7}{2}}}+\frac{R_{14}}{\alpha_{1}^{2}}+\frac{R_{15}}{\alpha_{1}}+R_{16}\right]^{-1}+O\left(\alpha_{1}^{2}\right) .
$$

The dimensionless time-averaged flux $\bar{Q}$ across one wavelength is

$$
\bar{Q}=\int_{0}^{1} \int_{0}^{h} r(w-1) d r d z=Q+\int_{0}^{1} h^{2} d z=Q+1+\frac{\varepsilon^{2}}{2} .
$$




\section{Pumping characteristics}

The pressure rise $(\Delta P)$ over one cycle of the wave is given by

$$
\Delta P=\int_{0}^{1} \frac{\partial p}{\partial z} d z
$$

The dimensionless frictional force $F$ at the wall across one wavelength is

$$
F=\int_{0}^{l} h^{2}\left(-\frac{\partial p}{\partial z}\right) d z
$$

\section{Results and discussion}

The present investigation focuses on the effects of various physiological parameters on the pressure rise $(\Delta P)$, pressure gradient $\left(\frac{\partial p}{\partial z}\right)$, frictional force $(F)$, temperature $(\theta)$ and streamlines $(\psi)$. Because of the complexity of the expression $\frac{\partial p}{\partial z}$, the expressions $\Delta P$ and $F$ are not integrable analytically. Thus, these quantities are numerically integrated with Weddle's rule using MATLAB for different values of physiological parameters and the results are plotted graphically in Figs 2-10.

Figure 2(i) is plotted to analyze the effects of $\tau$ on $\Delta P$. It is observed that with an increase in the values of $\tau, \Delta P$ increases in the pumping region $(\Delta P>0)$ and it decreases in the augmented region $(\Delta P<0)$. Also note that for a particular value of $\tau$ the pressure rise curve for $\alpha_{1}=0$ lies below the curve for $\alpha_{1}=0.1$ and the behavior is opposite when $\tau$ is minimum. The effects of $\varepsilon$ on $\Delta P$ and $\bar{Q}$ are presented in Fig.2(ii). It is noticed from the figure that an increase in the value of $\varepsilon$ increases $\Delta P$ in the pumping region. It is also observed that for a particular value of $\varepsilon, \Delta P$ curve for $\alpha_{1}=0.1$ lies above the curve for $\alpha_{1}=0$ and the situation reverses in the case of the augmented region. An increase in the values of $\alpha$ enhances $\Delta P$ in a porous tube (Fig.3(i)). The effects of $\beta$ on $\Delta P$ and $\bar{Q}$ show that $\Delta P$ increases for an increase in the angle $\beta$. This observation on $\beta$ is in good agreement with the results of Nagarani [12]. Also, it is observed that the $\Delta P$ curve for $\alpha_{1}=0.1$ lies above the curve for $\alpha_{1}=0$ and the opposite trend is noticed in the case of the augmented region (Fig.3(ii)). Figure 4 illustrates the effects of Da on $\Delta P$ and $\bar{Q}$. It is noticed that for an increase in the values of $\mathrm{Da}, \Delta P$ decreases in the pumping region. Further, for $\mathrm{Da}=0$, the $\Delta P$ curve for $\alpha_{1}=0.1$ lies below the curve for $\alpha_{1}=0$ and the situation reverses for $\mathrm{Da}=0.02$ and 0.04 . This is mainly because an increase in the values of Da increases the porosity of the wall and thus $\bar{Q}$ decreases. Thus, from Figs 2-4, one can observe that the pressure curve with variable viscosity always lies above the cure with constant viscosity. This observation is in concurrence with the results obtained by Hayat and Ali [29]. 

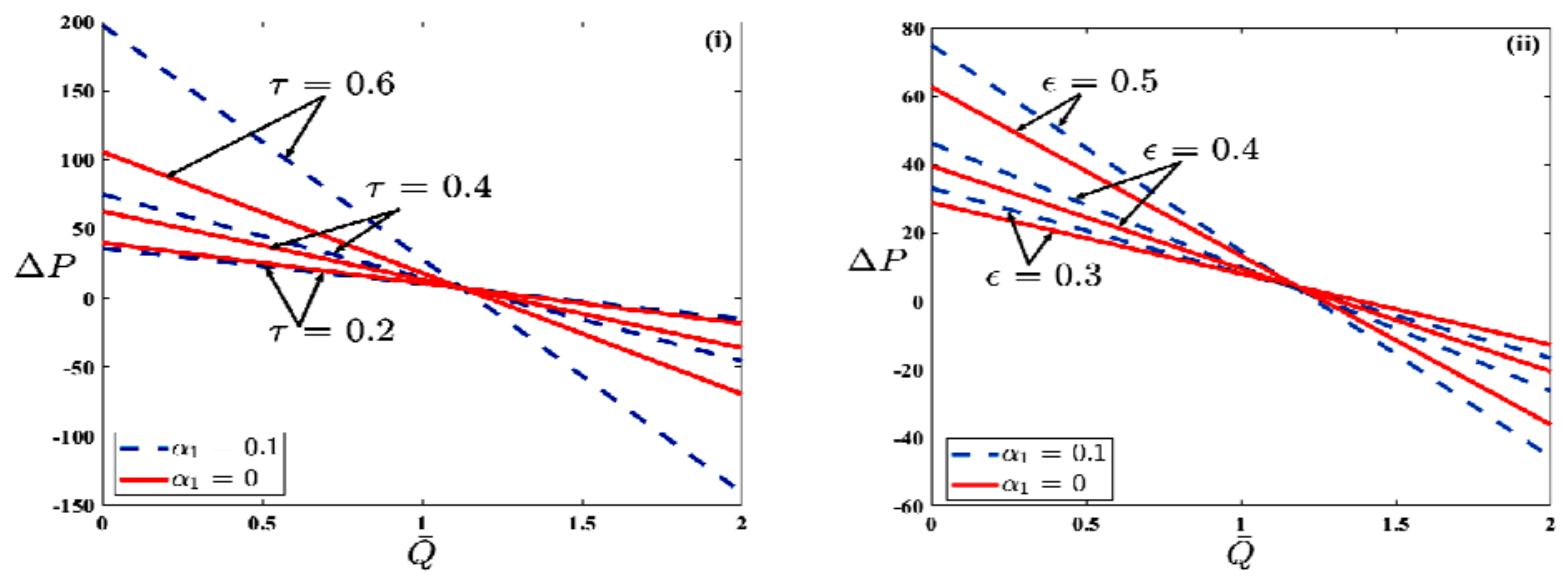

Fig.2. $\Delta P$ versus $\bar{Q}$ for varying (i) $\tau$ and (ii) $\varepsilon$ with $\mathrm{Da}=0.02, \alpha=0.2, \beta=\pi / 4$ and $F_{1}=0.1$.
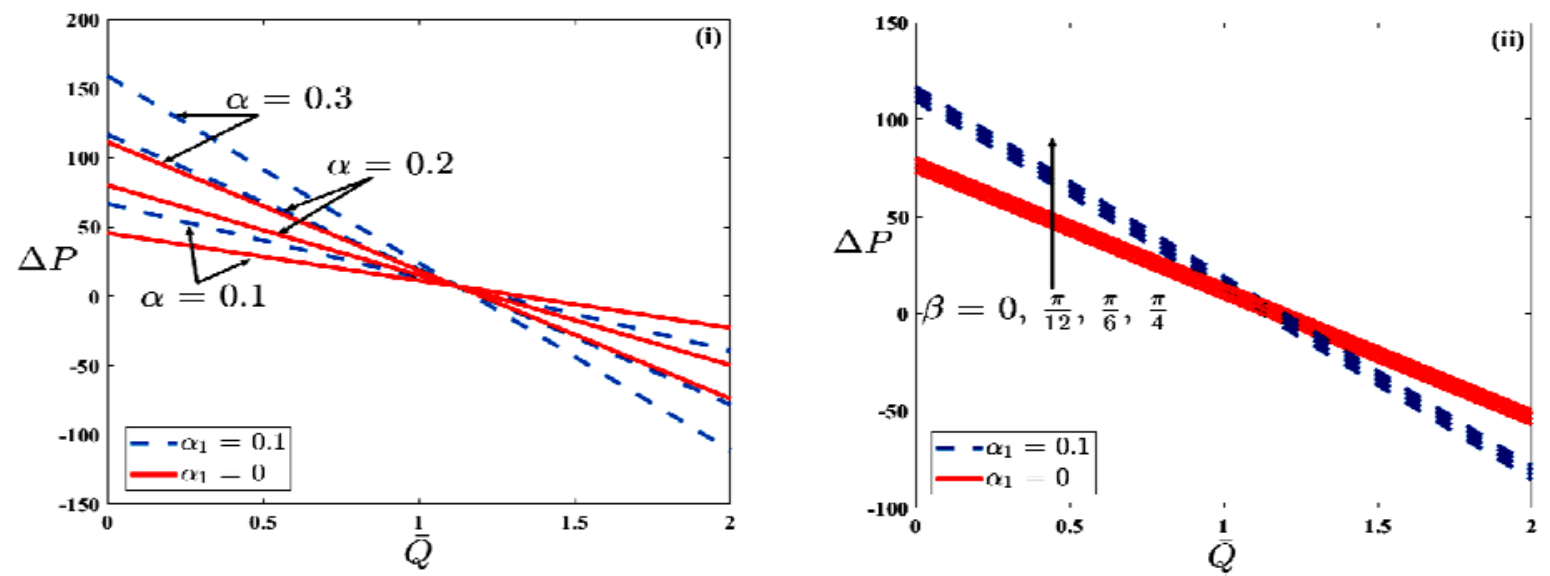

Fig.3. $\Delta P$ versus $\bar{Q}$ for varying (i) $\alpha$ and (ii) $\beta$ with $\tau=0.4, \varepsilon=0.5$, Da $=0.02$ and $F_{1}=0.1$.

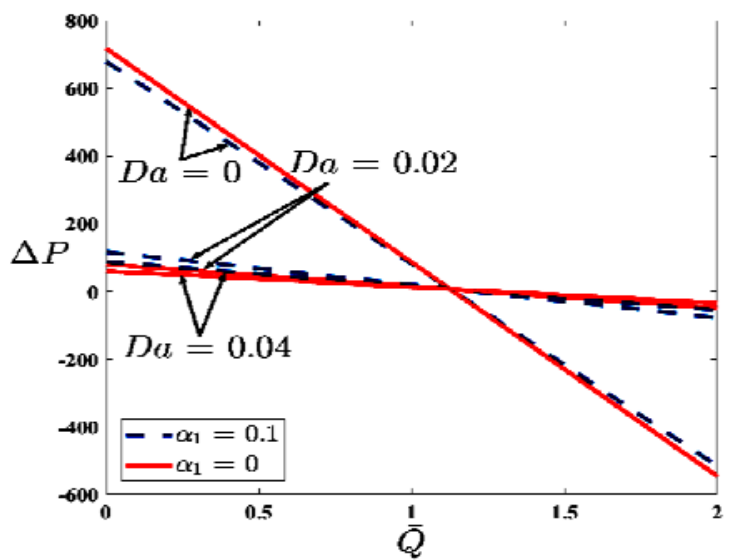

Fig.4. $\Delta P$ versus $\bar{Q}$ for varying Da with $\tau=0.4, \varepsilon=0.5, \alpha=0.2, F_{1}=0.1$ and $\beta=\pi / 4$. 
The above discussion regarding the effects of various physiological parameters is qualitative. To discuss the effects quantitatively, the intervals for $\bar{Q}$ where $\Delta P>0, \Delta P<0, F<0$ and $F>0$ are presented in Tabs 1-5. We observe that as $\tau$ increases the length of the interval for $\Delta P>0$ decreases (Tab.1). Also, for $\tau=0.2$ the length of the interval is more when $\alpha_{1}=0.1$ than $\alpha_{1}=0$ and for $\tau=0.4$ and 0.6 the opposite behavior is observed when viscosity increases from 0 to 0.1 . The effects of an increase in the values of $\varepsilon$ and $\mathrm{Da}$ decrease the length of the interval for $\Delta P>0$ and a similar observation is mode when viscosity increases from 0 to 0.1 (Tabs 2 and 3). The behavior of $\alpha$ and $\beta$ is opposite to that of $\varepsilon$ and Da (Tabs 4 and 5). However, from Tabs 1-5 it is noticed that the impact of $\tau, \varepsilon, \mathrm{Da}, \alpha$ and $\beta$ on frictional force shows the opposite trend as that of the pressure rise.

Table 1. Interval for time averaged flow rate $\bar{Q}$ across one wavelength for different values of $\tau$.

\begin{tabular}{|c|l|l|l|l|l|}
\hline \multicolumn{2}{|c|}{ Parameter : $\tau$} & $\begin{array}{c}\text { Interval for } \bar{Q} \\
\text { when } \Delta P>0\end{array}$ & $\begin{array}{c}\text { Interval for } \bar{Q} \\
\text { when } \Delta P<0\end{array}$ & $\begin{array}{c}\text { Interval for } \bar{Q} \\
\text { when } F<0\end{array}$ & $\begin{array}{c}\text { Interval for } \bar{Q} \\
\text { when } F>0\end{array}$ \\
\hline \multirow{2}{*}{0.2} & $\alpha_{1}=0$ & $0<\bar{Q}<1.367$ & $1.367<\bar{Q}<2$ & $0<\bar{Q}<1.788$ & $1.788<\bar{Q}<2$ \\
\cline { 2 - 6 } & $\alpha_{1}=0.1$ & $0<\bar{Q}<1.402$ & $1.402<\bar{Q}<2$ & $0<\bar{Q}<1.893$ & $1.893<\bar{Q}<2$ \\
\hline 0.4 & $\alpha_{1}=0$ & $0<\bar{Q}<1.268$ & $1.268<\bar{Q}<2$ & $0<\bar{Q}<1.516$ & $1.516<\bar{Q}<2$ \\
\cline { 2 - 6 } & $\alpha_{1}=0.1$ & $0<\bar{Q}<1.243$ & $1.243<\bar{Q}<2$ & $0<\bar{Q}<1.450$ & $1.450<\bar{Q}<2$ \\
\hline 0.6 & $\alpha_{1}=0$ & $0<\bar{Q}<1.206$ & $1.206<\bar{Q}<2$ & $0<\bar{Q}<1.346$ & $1.346<\bar{Q}<2$ \\
\cline { 2 - 6 } & $\alpha_{1}=0.1$ & $0<\bar{Q}<1.167$ & $1.167<\bar{Q}<2$ & $0<\bar{Q}<1.240$ & $1.240<\bar{Q}<2$ \\
\hline
\end{tabular}

Table 2. Interval for time averaged flow rate $\bar{Q}$ across one wavelength for different values of $\varepsilon$.

\begin{tabular}{|c|l|l|l|l|l|}
\hline \multicolumn{2}{|c|}{ Parameter : $\varepsilon$} & $\begin{array}{c}\text { Interval for } \bar{Q} \\
\text { when } \Delta P>0\end{array}$ & $\begin{array}{c}\text { Interval for } \bar{Q} \\
\text { when } \Delta P<0\end{array}$ & $\begin{array}{c}\text { Interval for } \bar{Q} \\
\text { when } F<0\end{array}$ & $\begin{array}{c}\text { Interval for } \bar{Q} \\
\text { when } F>0\end{array}$ \\
\hline 0.3 & $\alpha_{1}=0$ & $0<\bar{Q}<1.304$ & $1.304<\bar{Q}<2$ & $0<\bar{Q}<1.415$ & $1.415<\bar{Q}<2$ \\
\cline { 2 - 6 } & $\alpha_{1}=0.1$ & $0<\bar{Q}<1.221$ & $1.221<\bar{Q}<2$ & $0<\bar{Q}<1.298$ & $1.298<\bar{Q}<2$ \\
\hline 0.4 & $\alpha_{1}=0$ & $0<\bar{Q}<1.259$ & $1.259<\bar{Q}<2$ & $0<\bar{Q}<1.419$ & $1.419<\bar{Q}<2$ \\
\cline { 2 - 6 } & $\alpha_{1}=0.1$ & $0<\bar{Q}<1.201$ & $1.201<\bar{Q}<2$ & $0<\bar{Q}<1.310$ & $1.310<\bar{Q}<2$ \\
\hline 0.5 & $\alpha_{1}=0$ & $0<\bar{Q}<1.234$ & $1.234<\bar{Q}<2$ & $0<\bar{Q}<1.423$ & $1.423<\bar{Q}<2$ \\
\cline { 2 - 6 } & $\alpha_{1}=0.1$ & $0<\bar{Q}<1.198$ & $1.198<\bar{Q}<2$ & $0<\bar{Q}<1.327$ & $1.327<\bar{Q}<2$ \\
\hline
\end{tabular}


Table 3. Interval for time averaged flow rate $\bar{Q}$ across one wavelength for different values of Da.

\begin{tabular}{|c|l|l|c|c|c|}
\hline \multicolumn{2}{|c|}{ Parameter : Da } & $\begin{array}{c}\text { Interval for } \bar{Q} \\
\text { when } \Delta P>0\end{array}$ & $\begin{array}{c}\text { Interval for } \bar{Q} \\
\text { when } \Delta P<0\end{array}$ & $\begin{array}{c}\text { Interval for } \bar{Q} \\
\text { when } F<0\end{array}$ & $\begin{array}{c}\text { Interval for } \bar{Q} \\
\text { when } F>0\end{array}$ \\
\hline 0 & $\alpha_{1}=0$ & $0<\bar{Q}<1.137$ & $1.137<\bar{Q}<2$ & $0<\bar{Q}<1.155$ & $1.155<\bar{Q}<2$ \\
\cline { 2 - 6 } & $\alpha_{1}=0.1$ & $0<\bar{Q}<1.137$ & $1.137<\bar{Q}<2$ & $0<\bar{Q}<1.157$ & $1.157<\bar{Q}<2$ \\
\hline 0.02 & $\alpha_{1}=0$ & $0<\bar{Q}<1.234$ & $1.234<\bar{Q}<2$ & $0<\bar{Q}<1.423$ & $1.423<\bar{Q}<2$ \\
\cline { 2 - 6 } & $\alpha_{1}=0.1$ & $0<\bar{Q}<1.198$ & $1.198<\bar{Q}<2$ & $0<\bar{Q}<1.326$ & $1.326<\bar{Q}<2$ \\
\hline 0.04 & $\alpha_{1}=0$ & $0<\bar{Q}<1.275$ & $1.275<\bar{Q}<2$ & $0<\bar{Q}<1.534$ & $1.534<\bar{Q}<2$ \\
\cline { 2 - 6 } & $\alpha_{1}=0.1$ & $0<\bar{Q}<1.223$ & $1.223<\bar{Q}<2$ & $0<\bar{Q}<1.396$ & $1.396<\bar{Q}<2$ \\
\hline
\end{tabular}

The other parameters choosen are $\varepsilon=0.5, \alpha=0.2, \beta=\frac{\pi}{4}, \tau=0.5$ and $F_{1}=0.1$.

Table 4. Interval for time averaged flow rate $\bar{Q}$ across one wavelength for different values of $\alpha$.

\begin{tabular}{|c|l|l|l|l|l|}
\hline \multicolumn{2}{|c|}{ Parameter : $\alpha$} & $\begin{array}{c}\text { Interval for } \bar{Q} \\
\text { when } \Delta P>0\end{array}$ & $\begin{array}{c}\text { Interval for } \bar{Q} \\
\text { when } \Delta P<0\end{array}$ & $\begin{array}{c}\text { Interval for } \bar{Q} \\
\text { when } F<0\end{array}$ & $\begin{array}{c}\text { Interval for } \bar{Q} \\
\text { when } F>0\end{array}$ \\
\hline \multirow{2}{*}{0.1} & $\alpha_{1}=0$ & $0<\bar{Q}<1.332$ & $1.332<\bar{Q}<2$ & $0<\bar{Q}<1.690$ & $1.690<\bar{Q}<2$ \\
\cline { 2 - 6 } & $\alpha_{1}=0.1$ & $0<\bar{Q}<1.259$ & $1.259<\bar{Q}<2$ & $0<\bar{Q}<1.494$ & $1.494<\bar{Q}<2$ \\
\hline 0.2 & $\alpha_{1}=0$ & $0<\bar{Q}<1.234$ & $1.234<\bar{Q}<2$ & $0<\bar{Q}<1.423$ & $1.423<\bar{Q}<2$ \\
\cline { 2 - 6 } & $\alpha_{1}=0.1$ & $0<\bar{Q}<1.198$ & $1.198<\bar{Q}<2$ & $0<\bar{Q}<1.326$ & $1.326<\bar{Q}<2$ \\
\hline 0.3 & $\alpha_{1}=0$ & $0<\bar{Q}<1.202$ & $1.202<\bar{Q}<2$ & $0<\bar{Q}<1.334$ & $1.334<\bar{Q}<2$ \\
\cline { 2 - 6 } & $\alpha_{1}=0.1$ & $0<\bar{Q}<1.178$ & $1.178<\bar{Q}<2$ & $0<\bar{Q}<1.270$ & $1.270<\bar{Q}<2$ \\
\hline
\end{tabular}

Table 5. Interval for time averaged flow rate $\bar{Q}$ across one wavelength for different values of $\beta$.

\begin{tabular}{|c|l|l|l|l|l|}
\hline \multicolumn{2}{|c|}{ Parameter : $\beta$} & $\begin{array}{c}\text { Interval for } \bar{Q} \\
\text { when } \Delta P>0\end{array}$ & $\begin{array}{c}\text { Interval for } \bar{Q} \\
\text { when } \Delta P<0\end{array}$ & $\begin{array}{c}\text { Interval for } \bar{Q} \\
\text { when } F<0\end{array}$ & $\begin{array}{c}\text { Interval for } \bar{Q} \\
\text { when } F>0\end{array}$ \\
\hline 0 & $\alpha_{1}=0$ & $0<\bar{Q}<1.126$ & $1.126<\bar{Q}<2$ & $0<\bar{Q}<1.124$ & $1.124<\bar{Q}<2$ \\
\cline { 2 - 6 } & $\alpha_{1}=0.1$ & $0<\bar{Q}<1.126$ & $1.126<\bar{Q}<2$ & $0<\bar{Q}<1.124$ & $1.124<\bar{Q}<2$ \\
\hline$\pi / 12$ & $\alpha_{1}=0$ & $0<\bar{Q}<1.165$ & $1.165<\bar{Q}<2$ & $0<\bar{Q}<1.223$ & $1.223<\bar{Q}<2$ \\
\cline { 2 - 6 } & $\alpha_{1}=0.1$ & $0<\bar{Q}<1.152$ & $1.152<\bar{Q}<2$ & $0<\bar{Q}<1.198$ & $1.198<\bar{Q}<2$ \\
\hline \multirow{2}{*}{$\pi / 6$} & $\alpha_{1}=0$ & $0<\bar{Q}<1.202$ & $1.202<\bar{Q}<2$ & $0<\bar{Q}<1.335$ & $1.335<\bar{Q}<2$ \\
\cline { 2 - 6 } & $\alpha_{1}=0.1$ & $0<\bar{Q}<1.177$ & $1.177<\bar{Q}<2$ & $0<\bar{Q}<1.267$ & $1.267<\bar{Q}<2$ \\
\hline \multirow{2}{*}{$\pi / 4$} & $\alpha_{1}=0$ & $0<\bar{Q}<1.234$ & $1.234<\bar{Q}<2$ & $0<\bar{Q}<1.423$ & $1.423<\bar{Q}<2$ \\
\cline { 2 - 6 } & $\alpha_{1}=0.1$ & $0<\bar{Q}<1.198$ & $1.198<\bar{Q}<2$ & $0<\bar{Q}<1.326$ & $1.326<\bar{Q}<2$ \\
\hline
\end{tabular}

The other parameters choosen are $\varepsilon=0.5, \alpha=0.2, \mathrm{Da}=0.02, \tau=0.5$ and $F_{1}=0.1$. 
Figure 5 depicts the variation of $\tau$ and $\varepsilon$ on the pressure gradient. It is noticed from the figure that the magnitude of the pressure gradient increases with an increase in the values of $\tau$ (Fig.5(i)). Also, for $\tau=0.2$ the pressure curve for $\alpha_{1}=0.1$ lies below the curve for $\alpha_{1}=0$ and the situation reverses when $\tau=0.4$ and 0.6. A similar effect is observed for the variation of $\varepsilon$ on pressure (Fig.5(ii)). The effects of $\mathrm{Da}$ and $\beta$ decrease the magnitude of pressure in an inclined axisymmetric porous tube (Fig.6). The influence of $\alpha$ on pressure shows the opposite behavior as that of Da and $\beta$ (Fig.7). From Figs 5-7, it is seen that the maximum pressure gradient occurs when $z=0.75$ and the minimum pressure gradient exists near the walls of the tube. This confirms the fact that the fluid flow passes through a conduit comfortably in the middle.
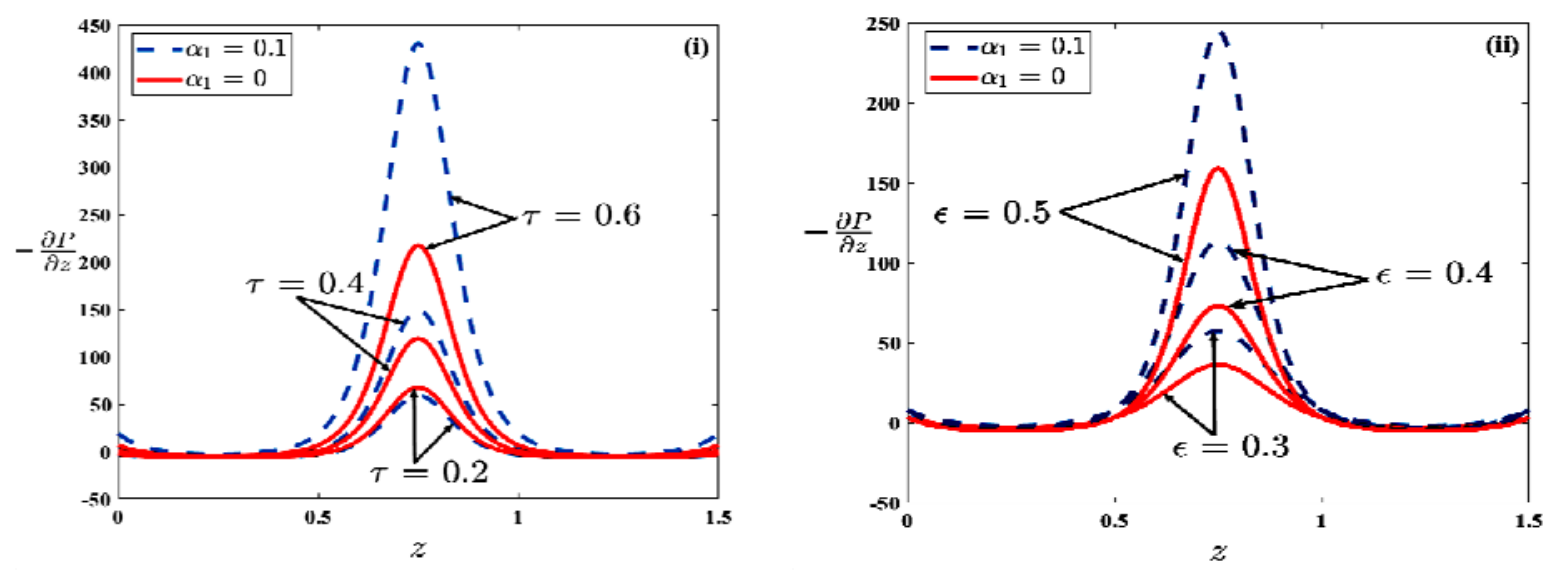

Fig.5. Pressure gradient versus $z$ for varying (i) $\tau$ and (ii) $\varepsilon$ with $\mathrm{Da}=0.02, \alpha=0.2, F_{1}=0.1$ and $\beta=\pi / 4$.
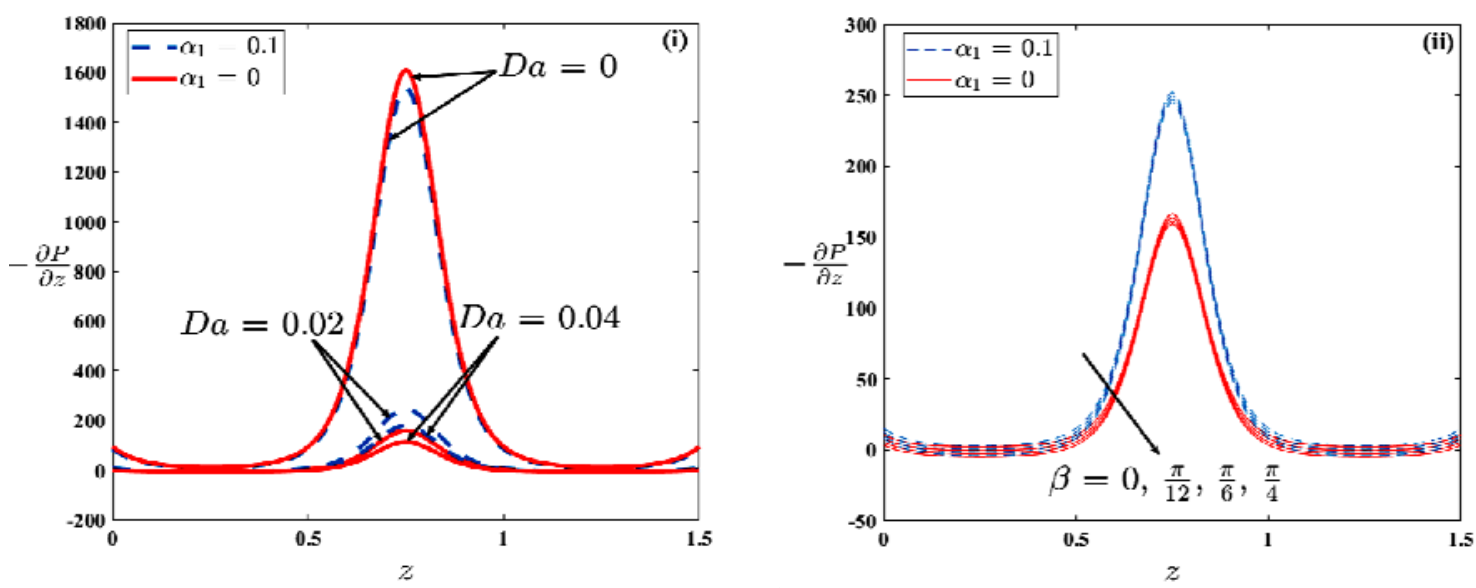

Fig.6. Pressure gradient versus $z$ for varying (i) $\mathrm{Da}$ and (ii) $\beta$ with $\tau=0.4, \varepsilon=0.5, \alpha=0.2$ and $F_{1}=0.1$. 


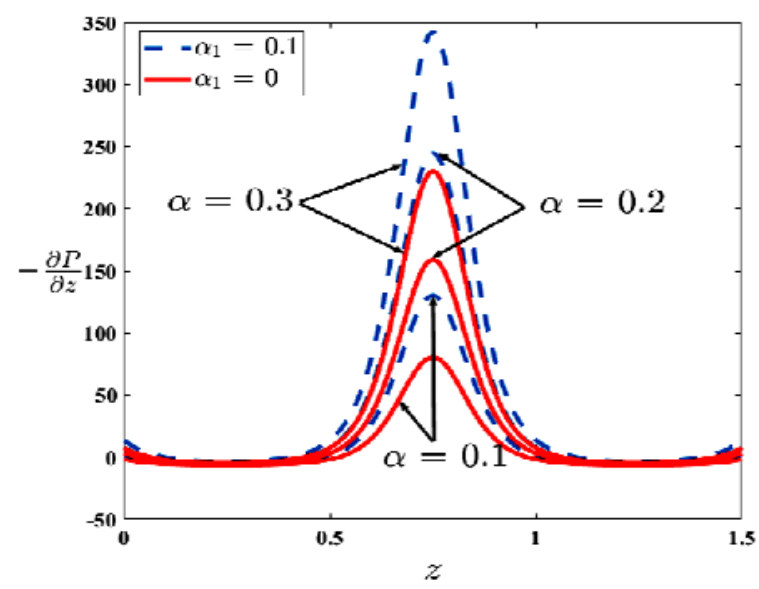

Fig.7. Pressure gradient versus $z$ for varying $\alpha$ with $\tau=0.4, \varepsilon=0.5, \mathrm{Da}=0.02, F_{1}=0.1$ and $\beta=\pi / 4$.

The variations of $F$ with $\bar{Q}$ with varying $\tau, \varepsilon, \alpha$ and $\beta$ are plotted in Figs 8 and 9. It is noticed that an increase in the values of $\tau, \varepsilon, \alpha$ and $\beta$ decreases the value of the frictional force. A similar behaviour is observed when the viscosity parameter increases from 0 to 0.1 . Also, the minimum frictional force is obtained at $\bar{Q}=0$ when $\tau, \varepsilon, \alpha$ and $\beta$ are maximum. Further, the effect of Da on $F$ shows the opposite behavior as that of $\tau, \varepsilon, \alpha$ and $\beta$ (See Fig.10).
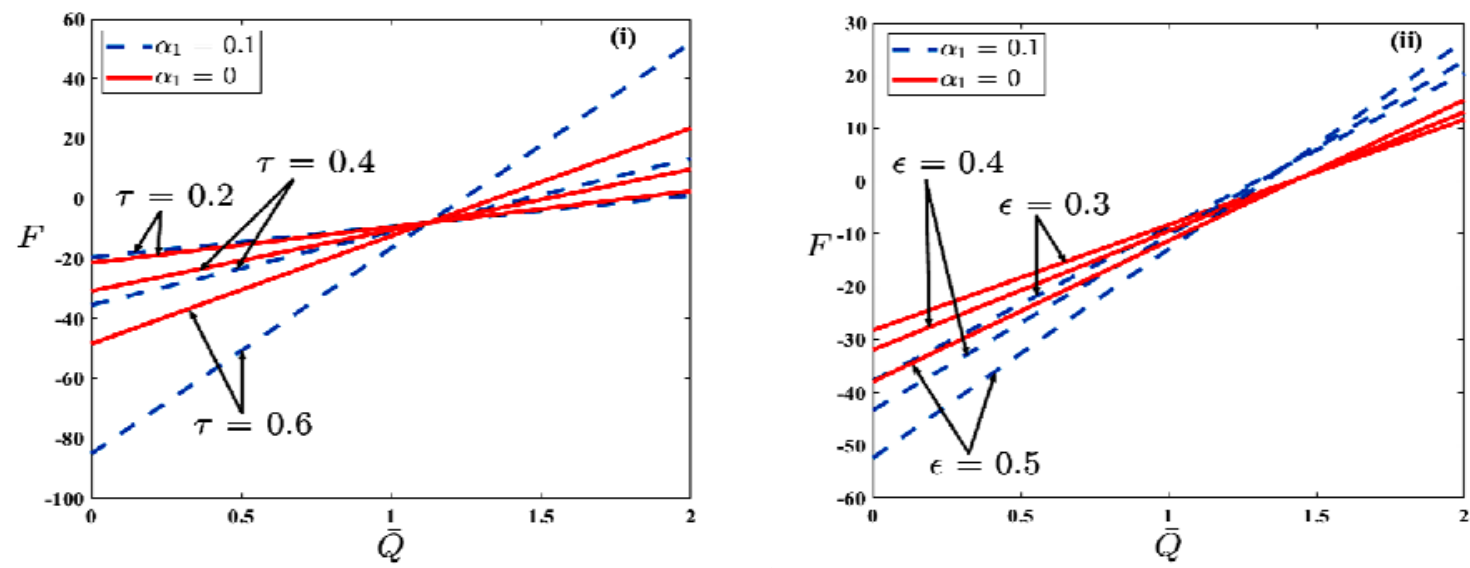

Fig.8. $F$ versus $\bar{Q}$ for varying (i) $\tau$ and (ii) $\varepsilon$ with $\mathrm{Da}=0.02, \alpha=0.2, F_{1}=0.1$ and $\beta=\pi / 4$. 

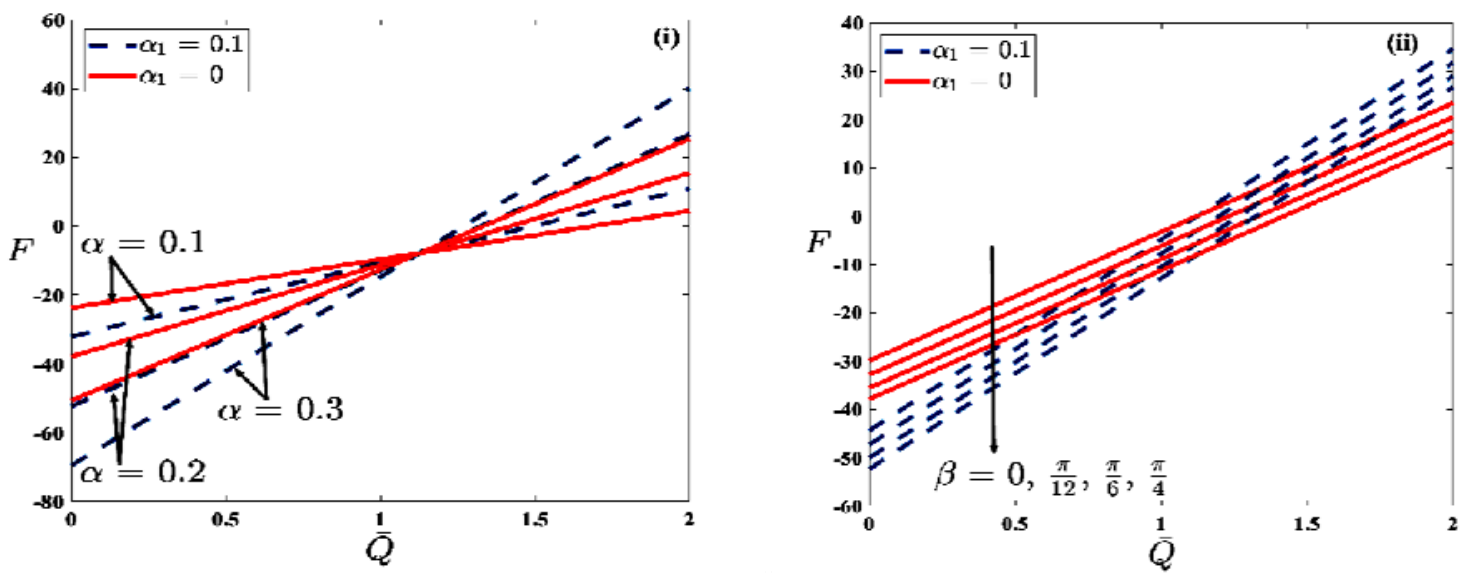

Fig.9. $F$ versus $\bar{Q}$ for varying (i) $\alpha$ and (ii) $\beta$ with $\tau=0.4, \varepsilon=0.5, \mathrm{Da}=0.02$, and $F_{1}=0.1$.

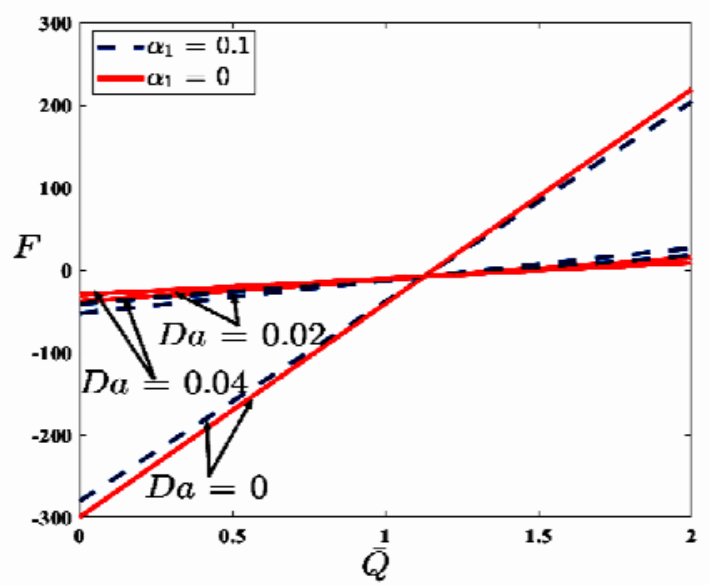

Fig.10. $F$ versus $\bar{Q}$ for varying Da with $\tau=0.4, \varepsilon=0.5, \alpha=0.2, \quad F_{1}=0.1$ and $\beta=\pi / 4$.

Figure 11 depicts the behavior of $\tau$ and $\varepsilon$ on $\theta$. It is observed that an increase in the values of $\tau$ and $\varepsilon$ increases $\theta$ in an inclined porous tube. Figure 12 illustrates the effect of the variation of $\alpha$ and Da on $\theta$. From Fig.12(i) it is noticed that an increase in the values of $\alpha$ significantly increases the temperature $(\theta)$. Further, the opposite behavior is observed in the case of Da (Fig.12(ii)). The variation of $\beta$ and $\gamma$ plays an important role in increasing $\theta$ (Fig.13). Figure 14 illustrates the impact of $\operatorname{Pr}$ and $\mathrm{Ec}$ on $\theta$. It is seen that an increase in Pr results in a decrease of $\theta$ (Fig.14(i)). Physiologically, an increase in Pr means a decrease in $k$ which is responsible for the decrease in $\theta$. Hence, cooling of the heated tube can be improved by choosing a coolant with a large Pr. Further, similar observations are made for an increase in the values of Ec (Fig.14(ii)). However, in all the cases $\theta$ increases when viscosity increases from 0 to 0.1 . Figures 15 and 16 represent the streamlines for different values of $\tau, \varepsilon$, Da and $\alpha$. It is observed that the volume of tapered bolus increases with an increase in the values of $\tau, \varepsilon$ and $\alpha$. Further, the size of tapered bolus decreases with an increase in the values of $\mathrm{Da}$. 

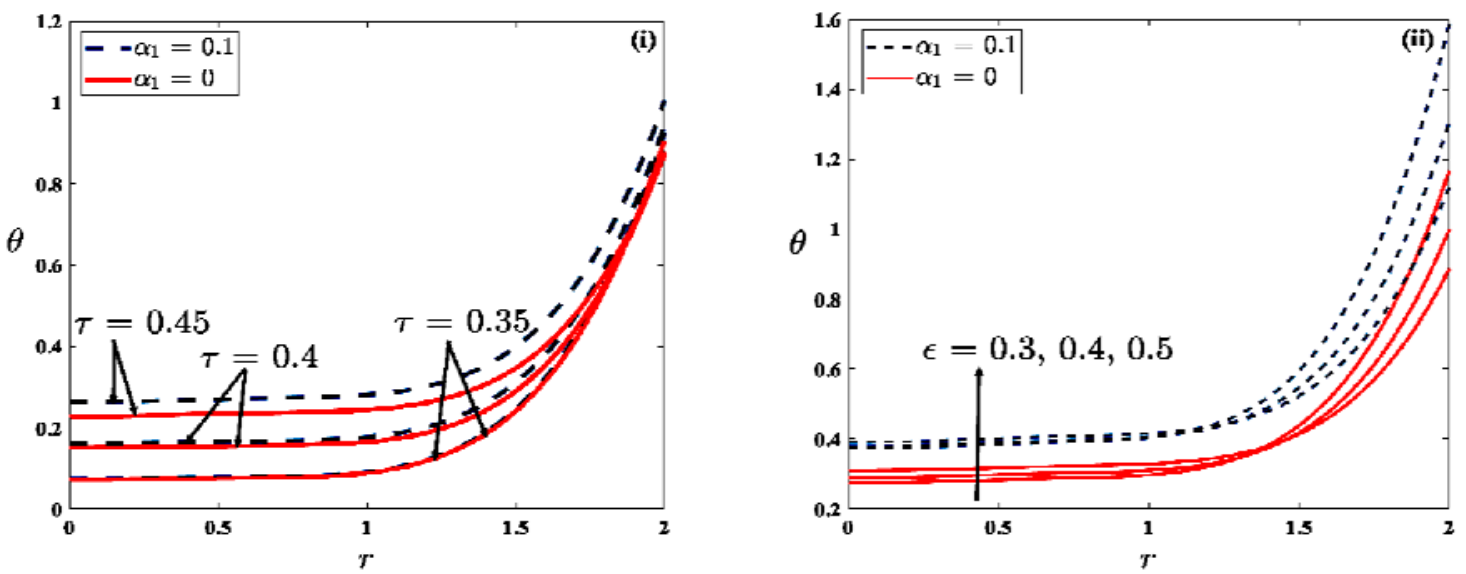

Fig.11. $\theta$ versus $r$ for varying (i) $\tau$ and (ii) $\varepsilon$ with $\alpha=0.2, \mathrm{Ec}=0.5, \operatorname{Pr}=0.5, \gamma=0.2, \mathrm{Da}=0.02, F_{1}=0.1$ and $\beta=\pi / 4$.
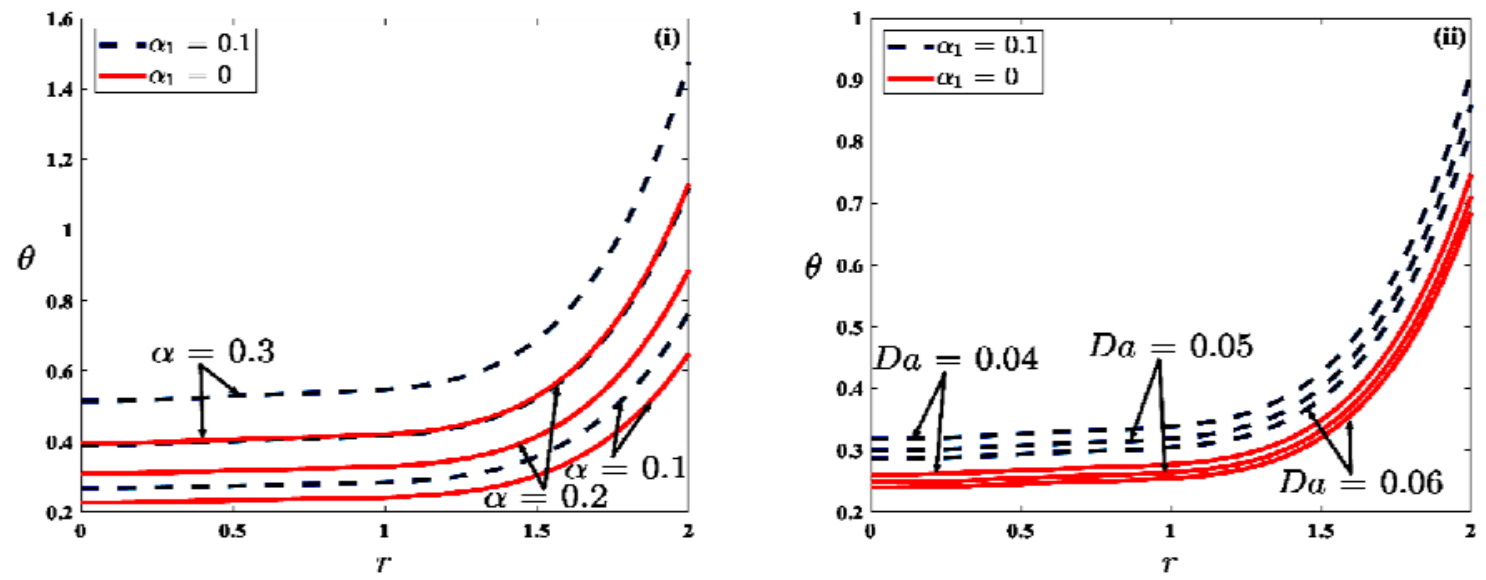

Fig.12. $\theta$ versus $r$ for varying (i) $\alpha$ and (ii) $\mathrm{Da}$ with $\tau=0.4, \mathrm{Ec}=0.5, \operatorname{Pr}=0.5, \gamma=0.2, \varepsilon=0.5$, $F_{1}=0.1$ and $\beta=\pi / 4$.
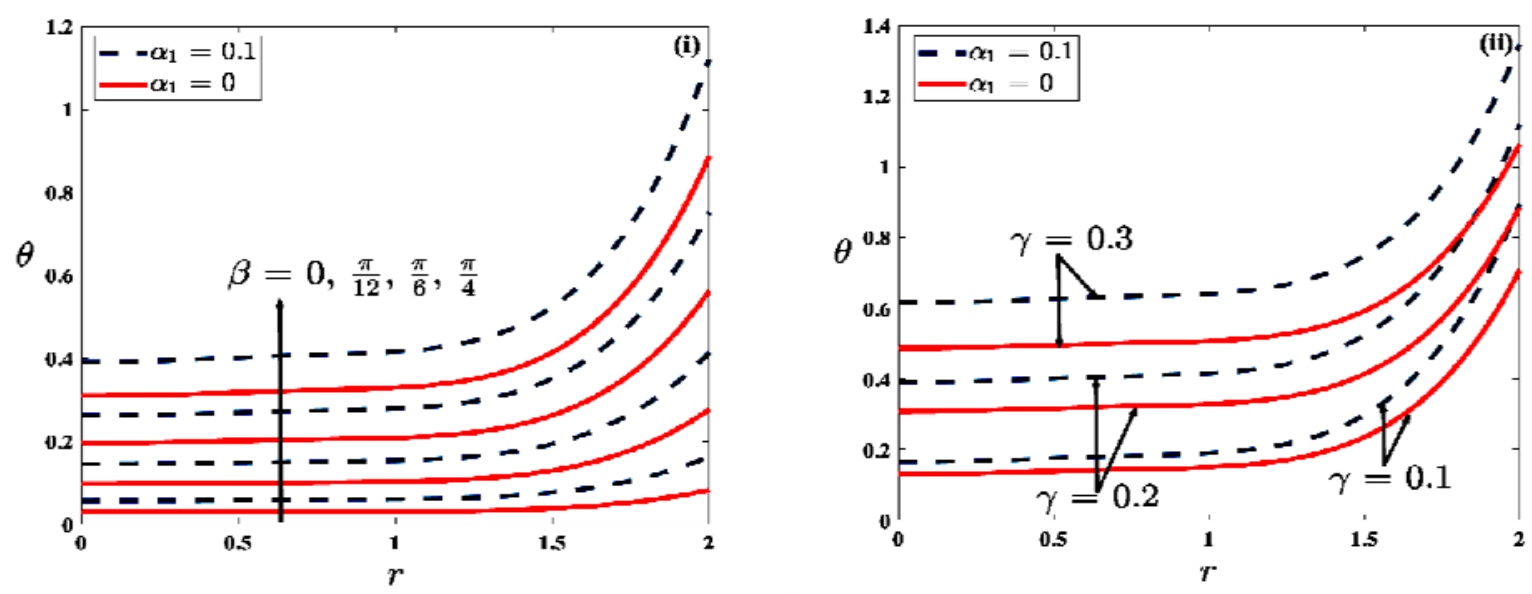

Fig.13. $\theta$ versus $r$ for varying (i) $\beta$ and (ii) $\gamma$ with $\mathrm{Da}=0.02, \tau=0.4, \mathrm{Ec}=0.5, \operatorname{Pr}=0.5, \alpha=0.2$, $\varepsilon=0.5$ and $F_{1}=0.1$. 

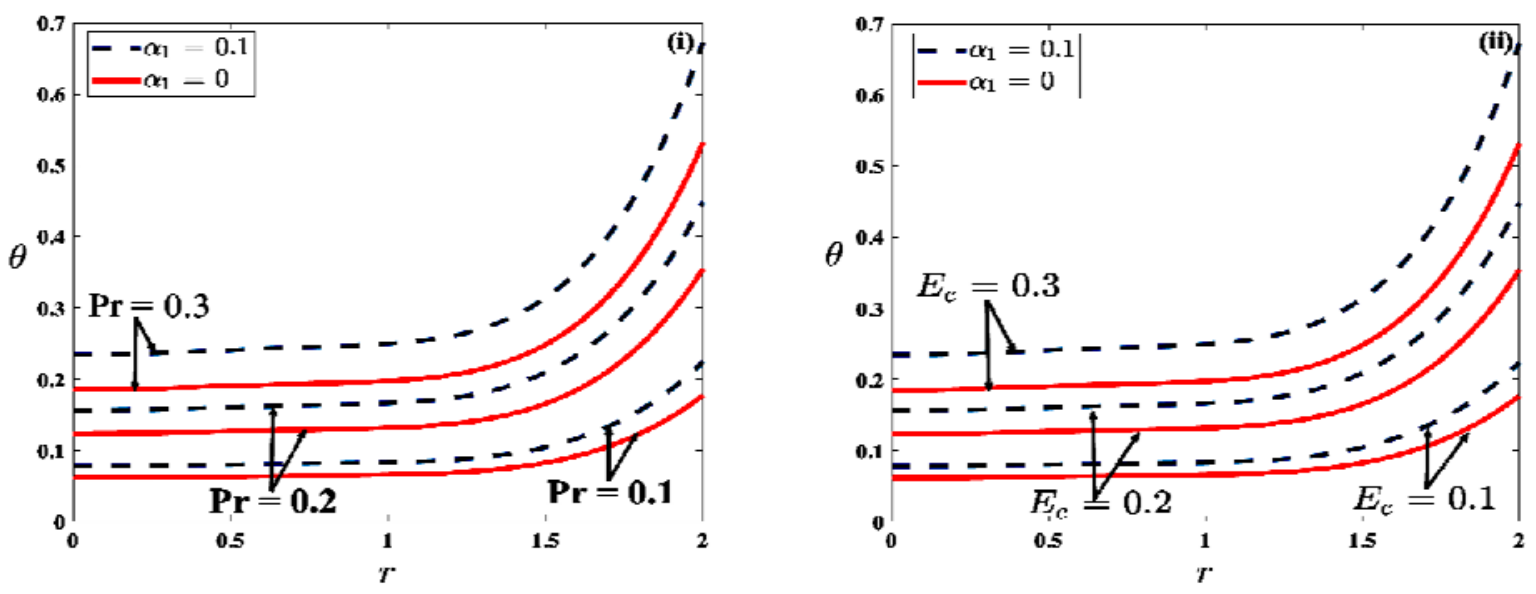

Fig.14. $\theta$ versus $r$ for varying (i) $\operatorname{Pr}$ and (ii) Ec with $\mathrm{Da}=0.02, \tau=0.4, \beta=\pi / 4, \alpha=0.2, \varepsilon=0.5$, $F_{1}=0.1$ and $\gamma=0.2$.
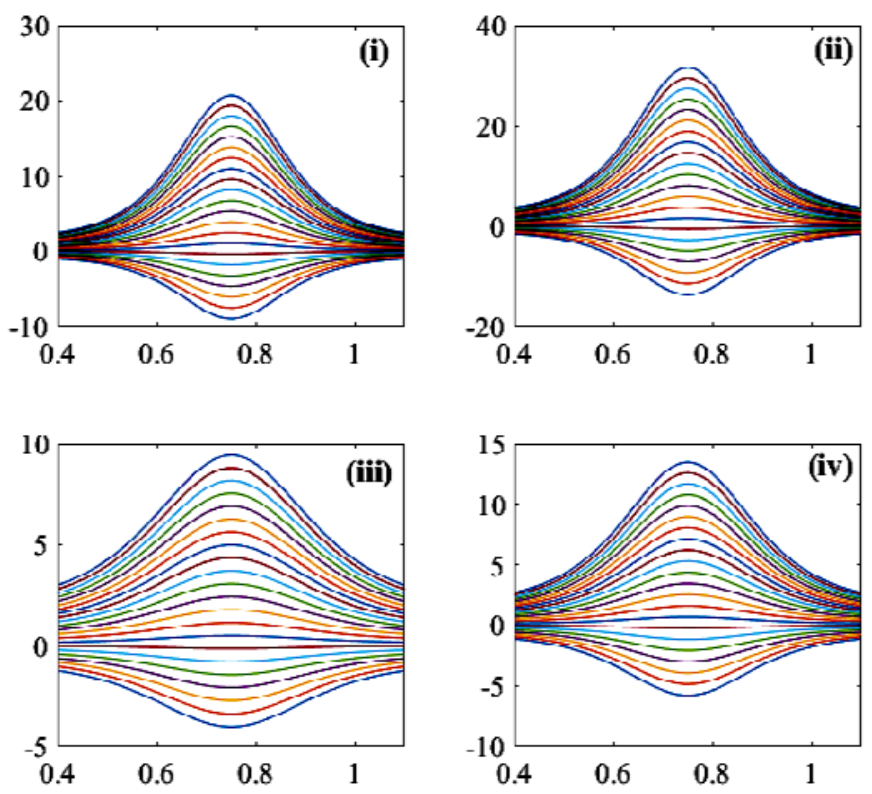

Fig.15. Streamlines for (i) $\tau=0.2$ and $\varepsilon=0.5$, (ii) $\tau=0.4$ and $\varepsilon=0.5$, (iii) $\varepsilon=0.3$ and $\tau=0.2$, (iv) $\varepsilon=0.4$ and $\tau=0.2$ with $\mathrm{Da}=0.02, \alpha=0.2, \alpha_{1}=0.1, \beta=\pi / 4$ and $F_{1}=0.1$. 

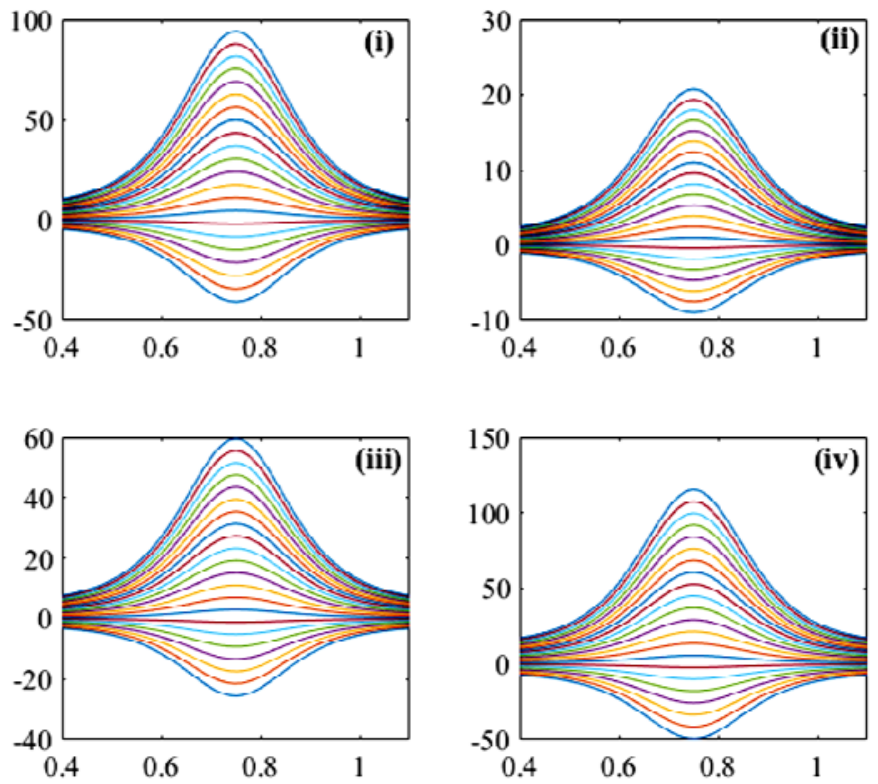

Fig.16. Streamlines for (i) $\mathrm{Da}=0$ and $\alpha=0.2$, (ii) $\mathrm{Da}=0.02$ and $\alpha=0.2$, (iii) $\alpha=0.3$ and $\mathrm{Da}=0.02$, (iv) $\alpha=0.4$ and $\mathrm{Da}=0.02$ with $\tau=0.4, \varepsilon=0.5, \alpha_{1}=0.1, \beta=\pi / 4$ and $F_{1}=0.1$.

\section{Summary and conclusions}

The present paper emphasizes the influence of varying viscosity, slip and heat transfer on peristaltic transport of the Casson fluid in an inclined porous tube. Also, from the current model, one can deduce the results for a Newtonian fluid in the absence of yield stress. The present study provides a satisfactory outcome that represents some of the natural phenomena, especially the flow of blood in narrow arteries which can be handled and processed in case of dysfunction. The conclusions can be summarized as follows:

$>$ it is possible to enhance the pressure rise, frictional force, temperature and peristaltic pumping performance by taking the variable viscosity into account;

$>$ the pumping performance increases with an increase in the slip parameter and decreases with an increase in porous parameter;

$>$ the pressure rise increases with increasing values of the yield stress, amplitude ratio and angle of inclination;

$>$ the magnitude of the pressure gradient is an increasing function of the yield stress, amplitude ratio and velocity slip parameter, and is a decreasing function of the Darcy number and angle of inclination;

$>$ the frictional force due to changes in the values of the yield stress, amplitude ratio, velocity slip parameter and angle of inclination decreases the frictional force and increases with increasing values of the Darcy number;

$>$ the temperature increases with an increasing amount of the yield stress, amplitude ratio, velocity slip parameter, thermal slip parameter, the angle of inclination, Eckert number and Prandtl number, and decreases with an increasing value of the Darcy number;

$>$ the volume of tapered bolus increases with an increase in the value of the yield stress, amplitude ratio and velocity slip parameter, and decreases as the Darcy number increases.

\section{Acknowledgement}

The authors appreciate the constructive comments of the reviewers which led to a definite improvement in the paper. 


\section{Appendix}

The expressions that appear in Section 3 are listed as follows

$$
\begin{aligned}
& R_{1}=-12 \alpha_{1} \sqrt{\alpha_{1} r_{p}}\left(2 r+\left\{-2+r_{p}\right\} r_{p}\right) \tanh ^{-1} \sqrt{\alpha_{1} h}, \\
& R_{2}=-2 \alpha_{1}^{3} \sqrt{\mathrm{Da}} h\left(2 r+\left\{-2+r_{p}\right\} r_{p}\right)\left(h+r_{p}-2 \sqrt{h r_{p}}\right), R_{3}=24 \alpha_{1} \sqrt{r r_{p}}, \\
& R_{4}=3 \alpha_{1} r^{2}+2 r\left[-3+\alpha_{1}\left\{12 \sqrt{h r_{p}}-8 \sqrt{r r_{p}}-3\left(h+r_{p}\right)\right\}\right], \\
& R_{5}=-18+\alpha_{1}\left[-3 h\left(-2+r_{p}\right)-24 \sqrt{h r_{p}}+16 r_{p}+3 r_{p}\left(1-3 r_{p}+4 \sqrt{h r_{p}}\right)\right],
\end{aligned}
$$$$
R_{6}=8 \alpha_{1} r \sqrt{\alpha_{1} r_{p}} \tanh ^{-1} \sqrt{\alpha_{1} r}-4\left(\alpha_{1} r_{p}\right)^{\frac{3}{2}} \tanh ^{-1} \sqrt{\alpha_{1} r_{p}}\left(2-r_{p}\right)
$$$$
R_{7}=8 \sqrt{\alpha_{1} r_{p}}\left[\tanh ^{-1} \sqrt{\alpha_{1} r_{p}}-\tanh ^{-1} \sqrt{\alpha_{1} r}\right], R_{8}=-\log \left(1-\alpha_{1} r\right)+\alpha_{1} r \log \left(\frac{\alpha_{1} r-1}{\alpha_{1} h-1}\right)+\log \left(1-\alpha_{1} r_{p}\right)
$$$$
R_{9}=\alpha_{1}\left(-2+r_{p}\right) r_{p} \log \left(\frac{\alpha_{1} r_{p}-1}{\alpha_{1} h-1}\right), R_{10}=30 \sqrt{\mathrm{Da}} h^{3}(h+\tau h-2 h \sqrt{2}), R_{11}=30 \alpha \log \left(\frac{1-\alpha_{1} \tau h}{1-\alpha_{1} h}\right)
$$$$
R_{12}=30 \alpha h\left[\sqrt{\tau}(4-3 \sqrt{\tau})+\tau \log \left(\frac{1-\alpha_{1} \tau h}{1-\alpha_{1} h}\right)-1\right], R_{13}=120 \alpha \sqrt{\tau h}\left[\tanh ^{-1} \sqrt{\alpha_{1} \tau h}-\tanh ^{-1} \sqrt{\alpha_{1} h}\right]
$$$$
R_{14}=\alpha h\left[10 h \sqrt{\tau}(4-3 \sqrt{\tau})+5 h r^{2}\left\{1+6 \log \left(\frac{1-\alpha_{1} h}{1-\alpha_{1} \tau}\right)\right\}-15 h\right]
$$$$
R_{15}=\alpha h\left[3 h^{2} \sqrt{\tau}(8-5 \sqrt{\tau})+h^{2} r^{3}\left\{1+30 \log \left(\frac{1-\alpha_{1} h}{1-\alpha_{1} \tau h}\right)\right\}-10 h^{2}\right]
$$

$$
R_{16}=\frac{30 \alpha h^{2} \tau^{2}}{\alpha_{1}} \log \left(\frac{\alpha_{1} \tau h-1}{\alpha_{1} h-1}\right)\left(\frac{1}{\alpha_{1}}+\tau h\right) .
$$




\section{Nomenclature}

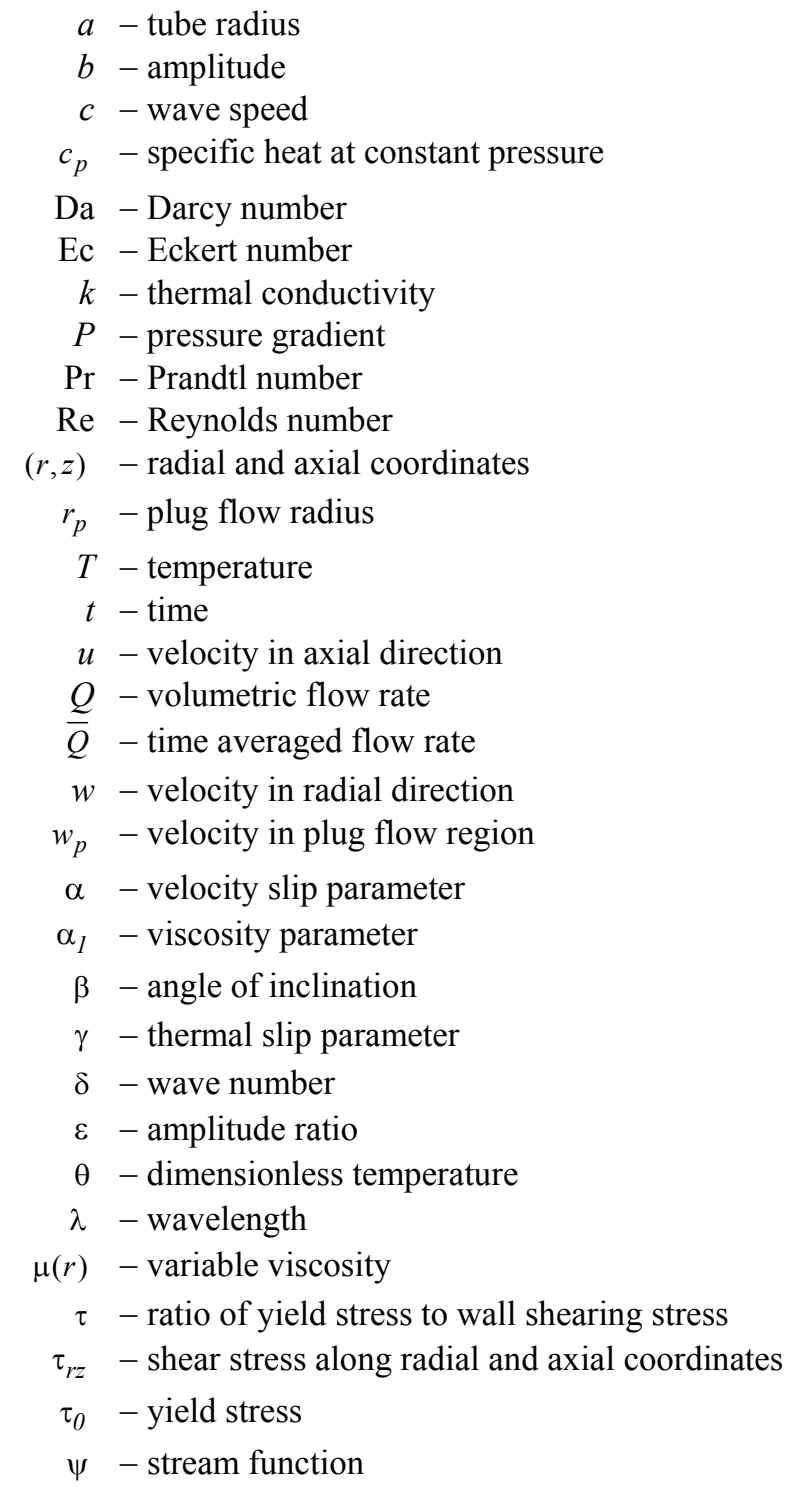

\section{References}

[1] Latham W. (1966): Fluid motions in the peristaltic pump. - M.S. Thesis, Boston: Massachusetts Institute of Technology.

[2] Burns J.C. and Parkes T. (1967): Peristaltic motion. - Journal of Fluid Mechanics, vol.29, pp.731-743.

[3] Shapiro A.H., Jaffrin M.Y. and Weinberg S.L. (1969): Peristaltic pumping with long wavelengths at low Reynolds number. - Journal of Fluid Mechanics, vol.37, pp.799-825.

[4] Rajashekhar C., Manjunatha G., Prasad K.V., Divya B.B. and Vaidya H. (2018): Peristaltic transport of twolayered Herschel Bulkley fluid. - Cogent Engineering, vol.5, pp.1495592.

[5] Elshehawey E.F., Mekheimer K., Kaldas S. and Afifi N. (1999): Peristaltic transport through a porous medium. - J. Biomath., vol.14. 
[6] Elshehawey E.F. and Husseny S.Z.A. (2000): Effects of porous boundaries on peristaltic transport through a porous medium. - Acta Mechanica, vol.143, pp.165-177.

[7] Vajravelu K., Sreenadh S., Lakshminarayana P., Sucharitha G. and Rashidi M.M. (2016): Peristaltic flow of Phan-Thein-Tanner fluid in an asymmetric channel with porous medium. - Journal of Applied Fluid Mechanics, vol.09, pp.1615-1625.

[8] Sreenadh S., Prasad K.V., Vaidya H., Sudhakar E., Gopi Krishna G. and Krinshnamurty M. (2016): MHD Couette flow of a Jeffrey fluid over a deformable porous layer. - Int. J. Appl. Comput. Math., vol.03, pp.21252138.

[9] Sankad G.C. and Nagathan P.S. (2017): Transport of MHD couple stress fluid through peristaltis in a porous medium under the influence of heat transfer and slip effects. - Int. J. of Applied Mechanics and Engineering, vol.22, No.2, pp.403-414.

[10] Ellahi R., Raza M. and Akbar N.S. (2017): Study of peristaltic flow of nanofluid with entropy generation in a porous medium. - Journal of Porous Media, vol.20, pp.461-478.

[11] Blair S.G.W. (1959): An equation for the flow of blood plasma and serum through glass capillaries. - Nature, vol.183, pp.613-614.

[12] Nagarani P. (2010): Peristaltic transport of Casson fluid in an inclined channel. - Korea-Australia Rheology Journal, vol.22, pp.105-111.

[13] Vajravelu K., Sreenadh S., Devaki P. and Prasad K.V. (2016): Peristaltic pumping of a Casson fluid in an elastic tube. - Journal of Applied Fluid Mechanics, vol.09, pp.1897-1905.

[14] Prasad K.V., Vajravelu K., Vaidya H., Shivakumara I.S. and Basha N.Z. (2016): Flow and heat transfer of a Casson Nanofluid over a nonlinear stretching sheet. - Journal of Nanofluids, vol.05, pp.743-752.

[15] Vajravelu K., Prasad K.V., Vaidya H., Basha N.Z. and Chiu-On-Ng. (2017): Mixed convective flow of a Casson fluid over a stretching sheet. - International Journal of Applied and Computational Mathematics, vol.03, pp.1619-1638.

[16] Chaturani P. and Narasimhan N. (1988): Theory for flow of Casson and Herschel-Bulkley fluids in coneplate viscometers. - Biorheology, vol.25, pp.199-207.

[17] Manjunatha G., Basavarajappa K.S., Thippeswamy G. and Vaidya H. (2013): Peristaltic transport of three layered viscous incompressible fluid. - Global Journal of Pure and Applied Mathematics, vol.09, pp.93-107.

[18] Manjunatha G. and Rajashekhar C. (2018): Slip effects on peristaltic transport of Casson fluid in an inclined elastic tube with orous walls. - Journa of Advanced Research in Fluid Mechanics and Thermal Sciences, vol.43, pp.67-80.

[19] Radhakrishnamacharya G. and Srinivasulu C. (2007): Influence of wall properties on peristaltic transport with heat transfer. - Comptes Rendus Mecanique, vol.335, pp.369-373.

[20] Srinivas S. and Kothandapani M. (2008): Peristaltic transport in an asymmetric channel with heat transfer a note. - International Communications in Heat and Mass Transfer, vol.35, pp.514-522.

[21] Nadeem S. and Akbar, N.S. (2009): Influence of heat transfer on a peristaltic transport of Herschel-Bulkley fluid in a non-uniform inclined tube. - Communication in Nonlinear Science and Numerical Simulation, vol.14, pp.4100-4113.

[22] Vajravelu K., Sreenadh S. and Lakshminarayana P. (2011): The influence of heat transfer on peristaltic transport of a Jeffery fluid in a vertical porous stratum. - Communications in Nonlinear Science and Numerical Simulation, vol.16, pp.3107-3125.

[23] Vajravelu K., Sreenadh S., Dhananjaya S. and Lakshminarayana P. (2016): Peristaltic flow and heat transfer of a conducting Phan-Thien-Tanner fluid in an asymmetric channel - Application to chime movement in small intestine. - Int. J. of Applied Mechanics and Engineering, vol.21, pp.713-736.

[24] Ramesh K. and Devakar, M. (2017): Influence of heat transfer on the peristaltic transport of Walters'B fluid in an inclined annulus. - Journal of the Brazilian Society of Mechanical Sciences and Engineering, vol.39, pp.2571-2584. 
[25] Hayat T., Zahir H., Alsaedi A. and Ahmad, B. (2017): Heat transfer analysis on peristaltic transport of ReeEyring fluid in a rotating frame. - Chinese Journal of Physics, vol.55, pp.1894-1907.

[26] Ebaid A., Emad H. A. and Vajravelu K. (2017): Analytical solution for peristaltic transport of viscous nanofluid in an asymmetric channel with full slip and convective conditions. - Communications in Theoretical Physics, vol.68, pp.96-102.

[27] Vaidya H., Manjunatha G., Rajashekhar C. and Prasad K.V. (2018): Role of slip and heat transfer on peristaltic transport of Herschel-Bulkley fluid through an elastic tube. - Multidiscipline Modelling in Materials and Structures, vol.14, pp.940-959.

[28] Devaki P., Sreenadh S., Vajravelu K., Prasad K.V. and Vaidya H. (2018): Wall properties and slip consequences on peristaltic transport of a Casson liquid in a flexible channel with heat transfer. - Applied Mathematics and Nonlinear Sciences, vol.3, pp.277-290.

[29] Hayat T. and Ali N. (2008): Effect of variable viscosity on the peristaltic transport of a Newtonian fluid in an asymmetric channel. - Applied Mathematical Modelling, vol.32, pp.761-774.)

[30] Lachiheb M. (2016): On the effect of variable viscosity on the peristaltic transport of a Newtonian fluid in an asymmetric channel. - Canadian Journal of Physics, vol.94, pp.320-327.

[31] Awais M., Bukhari U. Ali A. and Yasmin H. (2017): Convective and peristaltic viscous fluid flow with variable viscosity. - Journal of Engineering Thermophysics, vol.26, pp.69-78.

[32] Saffman P.G. (1971). On the boundary conditions at the surface of a porous medium. - Studies in Applied Mathematics, vol.01, pp.93-101.

[33] Bird R.B., Stewart W.E. and Lightfoot E.N. (1976): Transport Phenomena. - New York: Wiley.

Received: February 11, 2018

Revised: October 10, 2018 